\title{
Helical Liquids and Majorana Bound States in Quantum Wires
}

\author{
Yuval Oreg, ${ }^{1}$ Gil Refael, ${ }^{2}$ and Felix von Oppen ${ }^{3}$ \\ ${ }^{1}$ Department of Condensed Matter Physics, Weizmann Institute of Science, Rehovot, 76100, Israel \\ ${ }^{2}$ Department of Physics, California Institute of Technology, Pasadena, California 91125, USA \\ ${ }^{3}$ Dahlem Center for Complex Quantum Systems and Fachbereich Physik, Freie Universität Berlin, 14195 Berlin, Germany
}

(Received 16 March 2010; published 20 October 2010)

\begin{abstract}
We show that the combination of spin-orbit coupling with a Zeeman field or strong interactions may lead to the formation of a helical electron liquid in single-channel quantum wires, with spin and velocity perfectly correlated. We argue that zero-energy Majorana bound states are formed in various situations when such wires are situated in proximity to a conventional $s$-wave superconductor. This occurs when the external magnetic field, the superconducting gap, or, most simply, the chemical potential vary along the wire. These Majorana states do not require the presence of a vortex in the system. Experimental consequences of the helical liquid and the Majorana states are also discussed.
\end{abstract}

PACS numbers: 74.78.Na, 03.67.Lx, 73.63.Nm, 74.78.Fk

States of matter supporting Majorana fermions (MFs) have received much attention in the context of quantum computation. A widely separated pair of MF bound states forms a nonlocal fermionic state immune to local sources of decoherence, thus providing a platform for fault-tolerant quantum memory. Moreover, since MF states realize a representation of the non-Abelian braid group, topological quantum information processing can, in principle, be affected by braiding [1]. A realization of such states where they can be readily manipulated is therefore highly desirable.

There are several suggestions for physical systems that support MF states, for ways to measure their properties and manipulate them. These include fractional quantum Hall states at filling $\nu=5 / 2$ [2], $p$-wave superconductors [3], surfaces of $3 \mathrm{D}$ topological insulators proximate to a superconductor [4], superfluids in the ${ }^{3} \mathrm{He}-B$ phase [5,6], and helical edge modes of $2 \mathrm{D}$ topological insulators proximate to a ferromagnet and a superconductor [7]. Recently, it was suggested that a semiconducting thin film sandwiched between an $s$-wave superconductor and a magnetic insulator [8] will host MF states associated with superconducting vortices. All of these proposals are extremely challenging experimentally.

Realizing and manipulating MFs in wires may be decisively simpler. We show that quantum wires with strong spin-orbit coupling, e.g., InAs or InSb wires, and banded carbon nanotubes, form a helical liquid, akin to topological insulator edges. Consequently, these wires support MF states when in proximity to $s$-wave superconductors, and a magnetic field. Unlike their 2D counterparts, wire-MF states do not require the presence of a vortex in the system, eliminating decoherence arising from low lying vortexcore quasiparticle states. Most importantly, we explain how to produce and manipulate them by variations of a chemical potential, which could be simply produced by a set of micron-sized gates capacitatively coupled to the wire. Below we outline the key physical properties of MF states in quantum wires, their experimental signatures, and how to construct networks, enabling quantum information processing.

Without loss of generality, let the wire lie along the $y$ axis, the spin-orbit interaction, $u$, be along the $z$ axis, and a magnetic field $B$ be along the $x$ axis. Also, the wire is in contact with a superconductor, with proximity strength $\Delta$ (chosen real). The Hamiltonian is [4]

$$
\begin{aligned}
& H=\int \Psi^{\dagger}(y) \mathcal{H} \Psi(y) d y ; \quad \Psi^{\dagger}=\left(\psi_{\uparrow}^{\dagger}, \psi_{\downarrow}^{\dagger}, \psi_{\downarrow},-\psi_{\uparrow}\right) \\
& \mathcal{H}=\left[p^{2} / 2 m-\mu(y)\right] \tau_{z}+u p \sigma_{z} \tau_{z}+B(y) \sigma_{x}+\Delta(y) \tau_{x} .
\end{aligned}
$$

$\psi_{\uparrow,(\downarrow)}(y)$ annihilates spin-up (down) electrons at position $y$. The Pauli matrices $\sigma, \tau$ operate in spin and particle-hole space, respectively. $\mu$ is the chemical potential.

Zeeman field and superconducting proximity absent, the Hamiltonian (1) has an energy-momentum dispersion consisting of two shifted parabolas crossing at momentum $p=0$. The Zeeman field $B$ removes the level crossing and opens a gap at $p=0$. (Such a gap may also occur due to strong electron-electron interactions $[9,10]$, and therefore $B$ should be generally construed as either a magnetic field perpendicular to the spin-orbit coupling, or an interaction induced gap.) The pairing $\Delta$ opens a gap at the dispersion's outer wings (regardless of the Zeeman field's strength when strong spin-orbit coupling is present), which eliminates high-momentum excitations, thus leaving only the chiral states near $p=0$ as low energy excitation, which resemble the edge of a topological insulator $[4,10] . \Delta$ also affects these states, which allows us to tune the topological phase transitions essential for the production of MFs. Note that gapping out the highmomentum excitation can be done by coupling our system to an antiferromagnet with periodicity comparable to $2 k_{F}$, 
or using interactions to open a $\pm k_{F}$ pairing gap for $\mu$ away from the gap at $p=0$ [11].

The spectrum for constant $\mu, u, \Delta$, and $B$, is revealed by squaring $\mathcal{H}$ twice, which yields

$$
\begin{aligned}
E_{ \pm}^{2}= & B^{2}+\Delta^{2}+\xi_{p}^{2}+(u p)^{2} \\
& \pm 2 \sqrt{B^{2} \Delta^{2}+B^{2} \xi_{p}^{2}+(u p)^{2} \xi_{p}^{2}},
\end{aligned}
$$

where $\xi_{p}=p^{2} / 2 m-\mu$. Figure 1 shows examples of the spectrum. A linear vanishing and reopening gap when $B, \Delta, \mu$ vary indicates a topological phase transition. We denote the gaps near $p=0$ and near the Fermi momenta corresponding to $\xi_{p} \pm u p=0$ as $E_{0}$ and $E_{1}$, respectively.

The $p=0$ gap, $E_{0}$, is the key to the emergence of the MF states. Examining $E_{-}$at $p=0$ we notice that
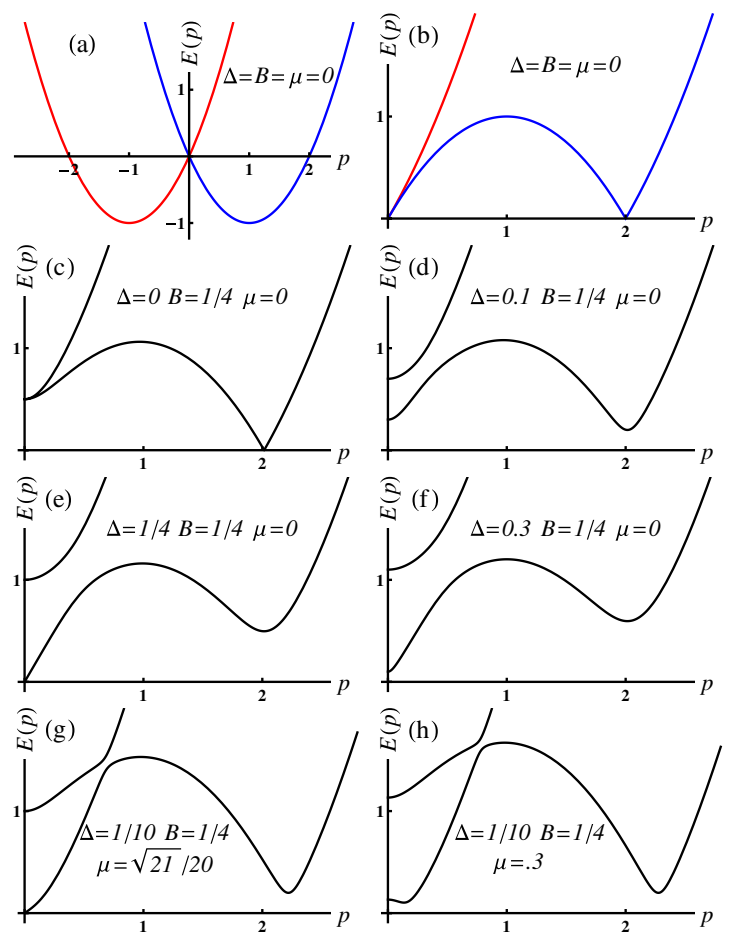

FIG. 1 (color online). (a) Single-particle spectrum for $\mu=$ $B=\Delta=0$. (The two shadings denote the different spin components.) The energy (momentum) scale is set by $m u^{2} / 2$ (by $m u$ ), with $u$ the spin-orbit coupling strength. (b) Excitation spectrum of adding or removing an electron for $\mu=B=$ $\Delta=0$. (c) Excitation spectrum for $B=1 / 4, \Delta=\mu=0$ where a gap due to the Zeeman term opens near $p=0$. (d) $B=2.5$, $\Delta=1 / 2, \mu=0$ with a superconducting gap in the wings and a $B$ dominated gap near the origin. This situation is analogous to a $p$-wave superconductor. We refer to this phase as the " $B$ dominated phase" (e) $B=1 / 4=\Delta=1 / 4, \mu=0$. The gap near $p=0$ closes, the gap at finite $p$ persists. At this critical point a quantum phase transition occurs. (f) $B=1 / 4, \Delta=0.3$, $\mu=0$. All gaps in the excitation spectrum are controlled by $\Delta$. (g) $B=1 / 4, \Delta=.1, \mu=\sqrt{B^{2}-\Delta^{2}}=\sqrt{21} / 20$. The gap at $p=0$ closes due to the shift in chemical potential. (h) A superconducting gap opens up in the entire spectrum due to the shift of the chemical potential above its critical value $\Delta=1 / 10$, $B=1 / 4, \mu=0.3$.

$$
E_{0}=E(p=0)=\left|B-\sqrt{\Delta^{2}+\mu^{2}}\right| .
$$

For $B^{2}>\Delta^{2}+\mu^{2}, \quad E_{0}$ is a $B$-dominated (or strong interaction induced) gap; the wire is in its topological phase, with MF states at the wire's ends [12]. When $B^{2}<$ $\Delta^{2}+\mu^{2}$ the gap is pairing dominated, with no end states; when $B^{2}=\Delta^{2}+\mu^{2}$ a quantum phase transition occurs. The gap $E_{1}$ near $p^{2}=2 \mu m$ remains a finite pairing gap throughout, since $\Delta$ always stays finite.

The phase transition evident in $E_{0}$ allows Majorana states to form. These can be achieved in various ways since $E_{0}$ depends on $B, \Delta$, and $\mu$. As in 2D topological insulator edges [4], a MF bound state forms when $B$ changes spatially and crosses $\Delta$, e.g., at $y=0$ [cf. Fig. 2(b)], or when $\Delta$ varies and crosses $B$ [cf. Fig. 2(d)].

Here we emphasize a third possibility: varying the chemical potential $\mu$. E.g., consider $B>\Delta$ so that for $\mu=0$ we have a $B$-dominated gap $E_{0}$. But when $\mu>$ $\sqrt{B^{2}-\Delta^{2}}$, the gap $E_{0}$, Eq. (3), is pairing dominated. Thus, we can form a MF state by tuning $\mu$ between these values [cf. Fig. 2(c)]. Notice that changes in $\mu$ hardly influence the gap $E_{1}$, so states $\approx \pm k_{F}$ do not play a role.

In $1 \mathrm{D}$ we can explore MF states formation where their wave functions can be obtained essentially exactly. Consider, e.g., a long ring with one conducting channel, in proximity to a superconductor and a Zeeman field, as in Fig. 2(a). Since the relevant momenta are near $p=0$, below we use the Hamiltonian linearized in that region:

$$
\mathcal{H}=u p \sigma_{z} \tau_{z}-\mu(y) \tau_{z}+B(y) \sigma_{x}+\Delta(y) \tau_{x} .
$$

This approximation requires $B \ll m u^{2}$. The MF states obtained below, however, are present regardless of the Zeeman splitting to spin-orbit coupling ratio.

Spatially varying B.-Assume $\Delta>0$ is constant, $\mu=0$, and that $B>\Delta$ for $y>0$ and $B<\Delta$ for $y<0$ [Fig. 2(b); the periodic boundary conditions require another point where $B=\Delta$ ]. Near the crossing point $y=0$, we write $B(y)=\Delta+b y$. Because of particle-hole symmetry, it is useful to square $\mathcal{H}$; In addition to the

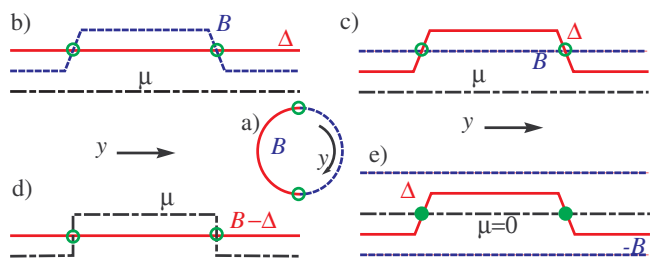

FIG. 2 (color online). (a) Wire in a ring geometry. Both halves have constant parameters and are joined by short junctions with a linearly varying parameter. MF states (marked by circles) are formed at the junctions. (b) MF state in the sector $p=0$ when $B$ varies. The gap in the finite- $p$ sector remains finite in the entire wire. (c) MF state in the sector $p=0$ when $\Delta$ varies. (d) MF state in the sector $p=0$ when $\mu$ varies. (e) " $p$ wave" MF state when $\Delta$ changes sign. The sector $p=0$ remains gapped in the entire wire. Each crossing with $\Delta=0$ hosts two MF states. 
squared, and the mixed $B \Delta$ terms, a term $\left\{u p \sigma_{z} \tau_{z}, B \sigma_{x}\right\}=$ $i \sigma_{y} \tau_{z} u[p, B]=\sigma_{y} \tau_{z} u b$ arises because $B$ depends on space and does not anticommute with the spin-orbit coupling term. Collecting all terms, we have

$$
\mathcal{H}_{b}^{2}=(u p)^{2}+B(y)^{2}+\Delta^{2}+u b \sigma_{y} \tau_{z}+2 \Delta B(y) \sigma_{x} \tau_{x} .
$$

Rotating $\mathcal{H}_{b}^{2}$ by $U_{b}^{\dagger}=1 / 2\left(\tau_{z}-i \tau_{x}-i \sigma_{x} \tau_{z}+\sigma_{x} \tau_{x}\right)$, we find that $U_{b} \mathcal{H}_{b}^{2} U_{b}^{\dagger}$ is diagonal with components $(u p)^{2}+$ $(\Delta \pm B)^{2} \pm u b$. The interesting modes are those with a minus sign in the brackets, $\Delta-B$. They correspond to an harmonic oscillator with ground-state wave function $\varphi(y)=\left(b /(u \pi)^{1 / 4}\right) e^{-b y^{2} /(2 u)}$ and energies $E_{n}^{2}=2 u b(n+$ $1 / 2) \pm u b, n=0,1,2, \ldots$ For $b>0$, the minus sign yields a zero-energy state with Bogoliubov operator

$\gamma_{b}^{\dagger}=\gamma_{b}=\frac{1}{\sqrt{2}}\left(\eta_{1}-\eta_{2}\right)=\frac{1}{2}\left(\psi_{\uparrow}-i \psi_{\downarrow}+i \psi_{\downarrow}^{\dagger}+\psi_{\uparrow}^{\dagger}\right)$,

$\eta_{1}=1 / \sqrt{2}\left(\psi_{\uparrow}^{\dagger}+\psi_{\uparrow}\right) ; \quad \eta_{2}=1 /(\sqrt{2} i)\left(\psi_{\downarrow}^{\dagger}-\psi_{\downarrow}\right)$.

The MF state at the second crossing point along the ring follows by $b \rightarrow-b$. Thus, this zero-energy state is $E_{0}^{+}=0$ with MF operator $-i / \sqrt{2}\left(\eta_{1}+\eta_{2}\right)$.

Spatially varying $\Delta$.- Here we assume $\Delta(y)=B+d y$, $\mu=0$, and $B$ constant [Fig. 2(c)]. The Hamiltonian here resembles the $y$-dependent $B$ case, if we exchange $\tau$ and $\sigma$ in Eqs. (4) and (5). Therefore, the MF states emerge here in exactly the same way as above, except with the diagonalizing matrices being $U_{d}^{\dagger}=U_{b}^{\dagger}(\tau \leftrightarrow \sigma)$, and $b \rightarrow d$ and $\Delta \rightarrow B$ in the resulting wave function. This yields (for positive $d$ ) $\gamma_{d}=\gamma_{d}^{\dagger}=\left(\eta_{1}-\eta_{2}\right) / \sqrt{2}$.

Spatially varying $\mu$.- If $B>\Delta$ everywhere, then at the interface between $B$-dominated regions with $\mu^{2}<B^{2}-\Delta^{2}$ and pairing gap regions with $\mu^{2}>B^{2}-\Delta^{2}$, a MF state also forms [Fig. 2(d)]. Here we assume that $\mu$ jumps abruptly at $y=0$ between $\mu_{\ell}$ for $y<0$, and $\mu_{r}$ at $y>0$. The condition for the MF state to form is

$$
\mu_{\ell}^{2}<B^{2}-\Delta^{2}, \quad \mu_{r}^{2}>B^{2}-\Delta^{2} .
$$

Matching the wave function at $y=0$ and using the ansatz $\psi_{r} \propto e^{-k_{r} y}$ for $y>0$ and $\psi_{l} \propto e^{k_{l} y}$ for $y<0$ we find

$$
\begin{aligned}
\mathcal{H}= & -\left(\Theta(y) k_{r}-\Theta(-y) k_{l}\right) i u \tau_{z} \sigma_{z}-\mu \tau_{z} \\
& +B \sigma_{x}+\Delta \tau_{x}=0
\end{aligned}
$$

where $u k_{r,(l) \pm}=\Delta \pm \sqrt{B^{2}-\mu_{(l) r}^{2}}$ and the eigenvectors

$$
\psi_{ \pm}^{r(l)}=e^{-(+) k_{r(l) \pm} y}\left(1, e^{ \pm i \theta_{r(l)}}, i,-i e^{ \pm i \theta_{r(l)}}\right)^{T} / 2
$$

with $\cos \theta_{r(l)}=\mu_{r(l)} / B$. One can easily verify that $\psi_{r(l)}$. $\Psi=\left(\psi_{r(l)} \cdot \Psi\right)^{\dagger}$ are MF operators, with $\psi$ a $c$ number. Thus, the wave function $\psi(y)$ of the MF state is

$$
\begin{aligned}
& 2 i \sin \theta_{r} \psi_{-}^{(0) \ell} \quad y<0 \\
& \left(e^{-i \theta_{l}}-e^{-i \theta_{r}}\right) \psi_{+}^{(0) r}+\left(e^{i \theta_{r}}-e^{-i \theta_{l}}\right) \psi_{-}^{(0) r} \quad y>0,
\end{aligned}
$$

exhausting all possibilities for isolated MF states.
We note that when $E_{0}$ is a $B$ dominated gap, the gap $E_{1}$ is due to pairing between spin-up electrons for positive $p$ and spin-down electrons for negative $p$, reminiscent of a onedimensional $p$-wave superconductor [13]. Recalling that vortices of a $p$-wave superconductor support a zero-energy bound state $[2,8,14]$, we expect the formation of MF states when $\Delta$ changes sign [Fig. 2(e)]. Because of the broken azimuthal symmetry, however, two inseparable MF states form where $\Delta$ vanishes.

Next we discuss experimental realizations. To be feasible, our proposal main requirement is a sufficiently strong spin-orbit interaction in a conducting single-channel wire weakly coupled to a superconductor. Previous experimental and the theoretical works describe different aspects of spin-orbit coupling in wires [15].

Several candidate systems for spin-orbit coupled wires exist. In carbon nanotubes, spin-orbit coupling arises due to curvature effects [16]. Here it is preferable to have a strong spin-orbit coupling along the direction of propagation, requiring that the tube is bent along its axis. Alternatively, one can use a strong electric field perpendicular to the axis. A more promising candidate is a wire of InAs in the wurtzite structure, known to have strong spinorbit coupling [17]. The velocity $u$ in the Hamiltonian equation (1) is related to the experimentally measured length scale $\lambda_{\mathrm{S} 0}=100 \mathrm{~nm}=m u$ and $\Delta_{\mathrm{SO}}=250 \mu \mathrm{V}=$ $m u^{2} / 2$ via $u \sim \hbar 2 \Delta_{\mathrm{SO}} \lambda_{\mathrm{SO}} \approx 7.6 \times 10^{6} \mathrm{~cm} / \mathrm{sec}$ and $m=\hbar^{2} / \lambda_{\mathrm{SO}}^{2} 2 \Delta=0.015 m_{e}$, with $m_{e}$ the free electron mass. Similar numbers (with $\Delta=280 \mu \mathrm{V}$ ) describe newly fabricated InSb wires, except with a large $g$ factor of $\sim 50$, compared to $g \sim 8$ in InAs, requiring only a small, relatively innocuous to the $\mathrm{SC}$, magnetic field [18].

Our wire-MF states can be formed by spatial variations of the Zeeman field, the proximity-induced superconductivity, or, most importantly, the chemical potential, and will form near points where $B^{2}-\left(\mu^{2}+\Delta^{2}\right)=0$. A varying chemical potential, as in Fig. 2(d), e.g., can be achieved by gate electrodes capacitatively coupled to the wire. Note that as long as the chemical potential gradients are slow compared to $\lambda_{\mathrm{SO}}$, the separate treatment of the large momentum wings, and linearization of the $p=0$ region are valid. Tunneling experiments should provide the most direct signatures of the MFs [19].

Additional experimental signatures arise by controlling the phase of the pairing $\Delta$ and the chemical potential. In particular, the configuration of Fig. 3 allows controlling $\Delta$ 's phase on the left $\left(\phi_{\ell}\right)$, center $\left(\phi_{c}\right)$, and right $\left(\phi_{r}\right)$ sections independently. The total Josephson current flowing between the three superconducting segments is rather intricate, and will be discussed in a separate publication. Since the MFs are localized when the distance between them, $L$, is infinite the Josephson current due to the MFs is zero. A straightforward first-order perturbation analysis for finite $L$ yields the energy splittings between the two MF states on the domain walls (cf. Ref. [20]). We find the Josephson energy associated with the MFs to be 


\section{$\Delta e^{i \phi_{l}} \quad B>\left|\Delta e^{i \phi_{c}}\right|$}

FIG. 3 (color online). $\mu, B$, or $\Delta$ are tuned to have a $B$-dominated gap at the wire's center region, while the other regions have a superconduting gap. The three disconnected superconducting electrodes in this configuration allow us to manipulate the phase difference in the two junctions separately.

$$
E=E_{\ell r} \cos \left(\frac{\phi_{\ell}-\phi_{r}}{2}\right)+E_{c} \cos \left(\frac{\phi_{\ell}+\phi_{r}}{2}-\phi_{c}\right)
$$

Here we assume that $\mu_{c}=0$ in the center region, and $\mu_{\ell}=\mu_{r}=\mu$ on the sides. Also, $E_{\ell r} \sim E_{c} \approx$ $\sqrt{\frac{2 \Delta B\left(\Delta^{2}-B^{2}\right)\left(\Delta^{2}+\mu^{2}-B^{2}\right)}{\Delta^{2}\left(\Delta^{2}+\mu^{2}-B^{2}\right)-B \mu^{2}(B+\Delta)}} e^{-(B-\Delta) L / u}$. In the similar setup of the edges of a topological insulator [21,22] the $E_{c}$, which is a result of the tunneling from the left and the right sections to the middle section is absent since the center region between the MFs is not in proximity to a superconductor. We notice two prominent effects. First, by letting $\phi_{c}=\phi_{r}$, for instance, we see that the Josephson current from the left superconductor is $4 \pi$ periodic. More interestingly, when we try to draw current from the center region, the current, proportional to $E_{c} \sin \left(\left(\phi_{\ell}+\phi_{r}-2 \phi_{c}\right) / 2\right)$ comes equally from the left and right superconductors. Therefore, this geometry can serve as a Josephson transistor, since a change of $\phi_{r}$ determines part of the current between the left and the middle section. Related effects were discussed in Ref. [23].

Next, we estimate the Josephson couplings in the setup of Fig. 3, with three superconducting substrates, connected only through the wire via proximity. The Josephson currents through the wire are given by Eq. (11), in addition to a contribution proportional to $\Delta$ and $2 \pi$ periodic in $\phi_{r}-\phi_{c}$ and $\phi_{\ell}-\phi_{c}$. In InAs wires, we expect the critical current for the $2 \pi$ periodic portion to be of order $40 \mathrm{nA}$, consistent with $B, \Delta \sim 1 \mathrm{~K}$. The $4 \pi$-periodic critical MajoranaJosephson currents, $2 e E_{c} / \hbar$ and $2 e E_{\ell r} / \hbar$, are a significant fraction of this number. For instance, for InAs parameters with $\mu_{c}=0$ and $\mu_{r}=\mu_{\ell}=0.9 B$ and $\Delta=0.8 B$, with $B \sim 1 \mathrm{~K}$, we obtain $E_{c} \approx 0.22 K e^{-L(B-\Delta) / u}$, corresponding to a maximum current $e E_{c} / \hbar \approx 4 n A \cdot e^{-L / 3 \mu \mathrm{m}}$ with $L$ the separation between the MFs. Low-frequency shot noise measurements would also reveal the anomalous Josephson periodicity of the Majorana-Josephson currents.

In summary, we have shown that wires with strong spin-orbit coupling in proximity to a superconductor host an interesting effective helical state. By tuning the superconducting gap $\Delta$, the Zeeman field $B$, or the chemical potential $\mu$, MF states can be created and detected in various experimental ways. By fabricating a set of gates over a network of wires, we can imagine adiabatically creating Majorana pairs, moving, and even interchanging them along the network using pulse sequences in the gates. Such networks will clearly display the Majorana's
non-Abelian character. Methods to manipulate the Majorana modes and their conductance signatures will be the subject of a future manuscript.

We would like to thank J. Moore, J. Alicea, E. Berg, and O. Starykh for enlightening discussions. The research was supported by ISF, DIP, BSF, and SPP 1285 grants, as well as the Packard and Sloan foundations, and by the Institute for Quantum Information under NSF Grants No. PHY0456720 and No. PHY-0803371.

Note added.-While finishing this manuscript we became aware of [24] which has some overlap with our results.

[1] C. Nayak et al., Rev. Mod. Phys. 80, 1083 (2008).

[2] G. Moore and N. Read, Nucl. Phys. B360, 362 (1991).

[3] T.M. Rice and M. Sigrist, J. Phys. Condens. Matter 7, L643 (1995).

[4] L. Fu and C. L. Kane, Phys. Rev. Lett. 100, 096407 (2008).

[5] G. E. Volovik, The Universe in a Helium Droplet (Oxford University Press, Oxford, 2003).

[6] M. A. Silaev and G. E. Volovik, arXiv:1005.4672.

[7] J. Nilsson, A. R. Akhmerov, and C. W. J. Beenakker, Phys. Rev. Lett. 101, 120403 (2008).

[8] J. D. Sau, R. M. Lutchyn, S. Tewari, and S. Das Sarma, Phys. Rev. Lett. 104, 040502 (2010).

[9] C. Xu and J. E. Moore, Phys. Rev. B 73, 045322 (2006).

[10] C. Wu, B. A. Bernevig, and S.-C. Zhang, Phys. Rev. Lett. 96, 106401 (2006).

[11] J. Sun, S. Gangadharaiah, and O. A. Starykh, Phys. Rev. Lett. 98, 126408 (2007).

[12] The end of the wire is characterized by a sharp drop of the chemical potential, thus closing the gap $E_{0}=$ $\left|B-\sqrt{\Delta^{2}+\mu^{2}}\right|$. This corresponds to a transition out of the $B$-dominated phase so that a Majorana fermion is localized near the end.

[13] Similar to the case of topological insulators the analogy can be made precise by a unitary transformation [4].

[14] N. Read and D. Green, Phys. Rev. B 61, 10267 (2000).

[15] A. V. Moroz, K. V. Samokhin, and C. H. W. Barnes, Phys. Rev. B 62, 16900 (2000); A. V. Moroz and C. H.W. Barnes, Phys. Rev. B 60, 14272 (1999); K. Hattori and H. Okamoto, Phys. Rev. B 74, 155321 (2006); J. Wan, M. Cahay, P. Debray, and R. Newrock, Phys. Rev. B 80, 155440 (2009).

[16] F. Kuemmeth, S. Ilani, D. C. Ralph, and P. L. McEuen, Nature (London) 452, 448 (2008).

[17] C. Fasth et al., Phys. Rev. Lett. 98, 266801 (2007).

[18] H. A. Nilsson et al., Nano Lett. 9, 3151 (2009).

[19] Y.E. Kraus, A. Auerbach, H. A. Fertig, and S. H. Simon, Phys. Rev. B 79, 134515 (2009).

[20] V. Shivamoggi, G. Refael, and J. E. Moore, Phys. Rev. B 82, 041405(R) (2010).

[21] L. Fu and C. L. Kane, Phys. Rev. Lett. 102, 216403 (2009).

[22] A. R. Akhmerov, J. Nilsson, and C. W. J. Beenakker, Phys. Rev. Lett. 102, 216404 (2009).

[23] Y. Tanaka, T. Yokoyama, and N. Nagaosa, Phys. Rev. Lett. 103, 107002 (2009).

[24] R. M. Lutchyn, J. D. Sau, and S. D. Sarma, Phys. Rev. Lett. 105, 077001 (2010). 